\title{
AN APPROACH FOR THE CORRELATION OF EMG SIGNALS AND THE RECTIFICATION OF PTB SOCKETS
}

\author{
N. A. ABU OSMAN* ${ }^{\dagger}$ and S. S. WONG \\ Department of Biomedical Engineering, Faculty of Engineering, \\ University of Malaya, 50603 Kuala Lumpur, Malaysia \\ *azuan@fk.um.edu.my \\ ${ }^{\dagger}$ Department of Bioengineering, University of Strathclyde, \\ Wolfson Building, 106 Rottenrow, Glasgow, G4 ONW, UK \\ ${ }^{\dagger}$ noor.abu-osman@strath.ac.uk \\ Z. OMAR \\ Faculty of Medicine, University of Malaya, \\ 50603 Kuala Lumpur, Malaysia \\ Received 4 April 2001 \\ Accepted 10 October 2001
}

\begin{abstract}
Prosthesis is often used to restore the appearance and functional mobility to individuals following limb amputatio $\mathrm{n}$. The purpose of this study is to investigate the correlation between the electromyogram (EMG) signals with the rectification of patellar tendon bearing (PTB) socket. Six PTB sockets are manufactured with different compression depths at the patellar tendon region. Experiments for the subject are carried out to obtain the EMG signals using EMG telemetry system. From this study, it is found that there exists some kinds of pattern as the depths of PTB rectification is changed. As the PTB rectification depth is increased, the EMG activity rises generally yet in an N pattern for the quadriceps group for both left and right legs of the amputee. The results of this case study will lead to an appropriate range of rectification depths for prosthetists in the manufacturing of PTB sockets, and perhaps the identification of the most suitable depth for optimum transtibial amputee ambulation.
\end{abstract}

Keywords: PTB socket; transtibial prosthesis; EMG signals; amputee gait; rehabilitation.

\section{Introduction}

The vertical support load is borne largely on the patellar-tendon - a 'tough' area well suited physiologically to this function. The pressure-tolerant areas include medial flare of the tibial tubercle, medial tibials plateau and posterior popliteal. According to Murdoch (1969), the custom practices, rectifying or deepening the socket at the patellar-tendon (which is between the distal end of the patellar and tibial tubercle) by about $3 / 4^{\prime \prime}$ or $18 \mathrm{~mm}$ (known as the neutral position). Studies by Abu Osman (2000) have shown that compression deeper than the neutral position as much as $5 \mathrm{~mm}$ can still be tolerated by the subject without any signs of discomfort, 
albeit it results in higher shear and normal stresses. This shows that the patellar tendon has the potential to bear more loads than what is normally supporting $(388.4 \mathrm{kPa}$ as compared to $245.1 \mathrm{kPa})$. It is found that much effort and expertise is spent on 3 broad fields: (1) the interface pressure distribution due to the rectification of PTB socket, (2) the EMG temporal analysis of gait, and (3) the gait analysis of transtibial amputee walking with PTB sockets.

\section{Literature Reviews}

Several groups such as Peat et al. (1995), Lee et al. (1997) has attempted to measure the interface shear stress on real subjects using strain gauge transducers. In particular, Abu Osman (2000) had rectified the patellar tendon region with compressions up to $5 \mathrm{~mm}$ from the neutral position (the original position as casted by the prosthetist) and measured the peak forces by using strain gauge custom-made technology. It was found that the patellar tendon bar is capable of bearing significantly more loads without any complaint or signs of discomfort from the subject. Peat et al. (1976), Winter and Sienko (1988) have carried out experiments on normal subjects to find out the phasic pattern of the various muscle activities. On the pathological gait analysis, Winter (1984) used computer-averaged EMG profiles to analyse spastic diplegia subjects. Peat et al. (1976) studied the telemetered EMG activity of lower extremity muscles on the affected side of 20 adult hemiplegic subjects. Winter and Sienko (1988) in his study of the biomechanics of below-knee amputee gait, used EMG profiles as one of the means to study the modified motor patterns from the residual muscles at the hip and knee.

This study aims to carry out a pilot study to obtain some quantitative EMG profiles with the variation of rectification depths.

\section{Methodology}

This study is carried out in 2 stages: (1) manufacture of 6 PTBs with varying rectification depths, (2) experimental and data processing.

\subsection{PTB Socket Manufacturing}

The PTB sockets are fabricated by an experienced qualified prosthetist. A total contact, supracondylar PTB socket rectified at the neutral position was custom made after castings have been made on the subject. Pelite foam is used as the material for inner liner and polypropylene as the outer socket. It serves as a model to fabricate other 5 experimental sockets, so that each socket would have uniform rectifications with only the PTB depth as a variable. The patellar tendon depth is rectified as required: compressed by 2, 4, $6 \mathrm{~mm}$; relief by $2,4 \mathrm{~mm}$. In order to measure this depth so as to satisfy the experimental socket requirement, a measurement gauge is designed. The prosthetist simply need to place the gauge on the 
rectification site. The plastic film will be the reference point from which depth will be measured by extending the pointer into the depth of the PTB region and the reading can be read off from the calibrated scale.

\subsection{Experimental and Data Processing}

This study was carried out in the Motion Analysis Laboratory, which is located at the Biomedical Engineering Program, Faculty of Engineering, University of Malaya. The laboratory is equipped with 4 subsystems: Kistler forceplates, Mespec 4000 8-channel EMG telemetry unit, videotaping system with 4 CCTV cameras and Peak Motus motion analysis software. The amputee subject was a female, aged 58 with a standard stump. She was asked to walk along a 5-m walkway in the experimental PTB socket at her natural cadence. The foot assembly used is an endolite multiflex foot and ankle attached to the PTB with a 30-mm diameter carbon fiber tube. Reflective markers were located at the following anatomical landmarks: heel, toe (fifth metatarsal), ankle, knee (lateral epicondyle), hip (greater trochanter) and sacrum. 8 EMG surface electrodes were pasted to the muscles of interest to record the muscle activities. These muscles are: gluteus maximus, vastus lateralis, rectus femoris and biceps femoris. These electrodes are attached to a telemetry bag, which has an antenna to transmit the signals to the receiving unit. Camera images taken at 50 frames per second were synchronized with the EMG and forceplate data. The forceplate data were normalised and plotted in percent time from the first right heel strike to the first left toe off. The raw EMG data were rectified and filtered with Butterworth filter cut off at 4-6 Hz. The resulting linear envelope for each trial of the experiments was compared and trials with similar pattern are averaged. This techniques also been used by other researchers, such as Winter and Sienko (1988), Vaughn et al. (1992), and Synder et al. (1995). For the EMG of the subject wearing PTB at different rectification depths, the data is quantified by summing the area under curve using the trapezoidal rule. Finally, a graph, which plots the area under curve against the PTB rectification depth, gives a clear indication of the effect of this varying parameter on the muscle activities.

\section{Results and Discussion}

\subsection{Ground Reaction Force (GRF)}

From the experiments, i.e. the vertical GRF, Fz shows great asymmetry on the left and right stance phase. The characteristic double peak of a normal $\mathrm{Fz}$ is absent, especially on the left stance phase. Moreover, the left stance phase remains longer on the ground than the right leg. This will cast a larger amount of body weight (BW) to be transferred on to the left side. The steeper slope on the left stance shows that the leg accelerates to a maximum of $108 \%$ BW and then the force flattens off for about $50 \%$ time. This shows that the loading on the prosthetic stance limb is significantly reduced compared to the loading on the intact limb. It seems that the amputee is 
trying to minimize the time spent on the prosthetic limb. Vaughn et al. (1992) shows this may be due to decreased proprioceptive feedback, a compensation for muscular deficiencies and/or a result of pain and discomfort experienced during walking.

The pattern and magnitude of the medial lateral GRF measured from the amputee subject also exhibits great deviations from the normal subject. The lateral reaction force is prolonged and decreasing in magnitude. As for the medial reaction force, the double hump as recorded in normal subjects, is distorted to become a single hump for an amputee. This reflects the inability of the amputee to swing the line of action of the resultant force inward to the left during right stance phase. Hence, there may be balancing problem during this phase. This shows that the right amputated leg is painful and unable to endure the load transfer as efficiently as the left leg.

$F x$, anterior/posterior force reveals a much less magnitude of braking and propulsion on the right as compared to the left. This is again, inevitable since the loss of right leg will cause insufficient proprioceptive control over the prosthesis to brake and propel as evenly and naturally as the left leg. As a consequence, the left leg will bear the major responsibility to control the forward and backward movement during gait.

\subsection{Normal and Amputee EMG Signal}

The amputee EMG is hyperactive compared to a normal subject. This due to the fact that muscles of the unilateral amputee have to work more in order to achieve the same motion as the normal subject due to the loss of a limb. The hip extensor was dominant for most of the stance phase during initial weight-bearing and continued past mid-stance. This resulted in above normal energy generation by the concentrically contracting the muscles, which is compensation made up for the loss of major energy generation by the plantar flexors at push-off. However, in both cases, the activity was below normal during the right heel strike (RHS). This may be because of the inability of the hip extensors to dorsiflex the prosthetic ankle at RHS.

Due to the hyperactivity of the hamstrings, there is an excessive knee flexor moment, which is cancelled out by the co-contracting knee extensors. The activity of gluteus maximus was dominant from left heel strike (LHS), peaked at the left mid-stance and then drop at the left toe off (LTO). This agrees with the apparent weight bearing on the good leg by the hip extensors. The left biceps femoris is hyperactive especially during mid-swing until LHS. This is to flex the knee during midstance. At left early and midstance, the action of the left biceps femoris (as the knee flexor) is cancelled out by the co-contraction of the rectus femoris and vastus lateralis. As a result, there is a net knee extensor moment generated.

The left quadriceps between the normal and amputee subjects show hyperactive during LBDS from LHS to RTO. This correlates well with the function of the quadriceps in braking mechanism mentioned previously. They worked eccentrically to control the knee from continuing flexing during the LBDS. 


\subsection{The Comparison of the PTB Rectification on EMG}

The comparison of EMG of the 8 muscles with the subject wearing PTB at various rectification depths, similar muscle firing patterns are observed and nonetheless, it has variations in terms of peaks. They have similar muscle activities for all the PTB sockets, which peaks at the right mid and late stance. But the magnitude of the curve is different for each $\mathrm{PTB}$ rectification. $\mathrm{PTB}+6$ has the highest peak, followed by $\mathrm{PTB}+4$, PTB neutral, $\mathrm{PTB}-2$ and $\mathrm{PTB}-4$. It also has the general muscle-firing pattern, but for different PTBs, the curves have different magnitudes at the peak. A technique called area under curve, or integration, is used to obtain a quantitative data about the activity of the muscles. The curve of for integration is from the first RHS to the first LTO. Using this data, then plot a graph of area under curve of the EMG vs. different PTB rectification depths. In doing so, it is manage to compare and see the differences in EMG by looking at the magnitude of EMG generated by wearing different PTB sockets.

\subsection{The Area Under EMG Curve}

As hypothesized in this study, there is a pattern, or correlation between the EMG and PTB rectification. Shown in Figs. 1 and 2 are the graphs of the left and right quadriceps muscles, which is of utmost importance, because it has common insertion at the patellar tendon into the tibial tubercle. It is observed that all the quadriceps group has similar pattern when the PTB worn are of different rectification depths, both on the left and right side. It looks like when rectification is increased (compressed), the EMG at the quadriceps will be increased. This is proven on the graphs at the rectification of $+6 \mathrm{~mm}$, the area under curve is always higher than the area under curve at $+4 \mathrm{~mm}$. The pattern in Figs. 1(a) and 1(c) can be explained by the fact that the deeper the rectification, the harder is the patellar tendon being compressed. Thus, this affects the attached muscles directly, which is the quadriceps. Higher activity is produced due to pain and discomfort.

The left quadriceps [Figs. 1(b) and 1(d)] also records similar pattern, indicating that the increase of rectification depth from $+4 \mathrm{~mm}$ to $+6 \mathrm{~mm}$ also causes the EMG on the left quadriceps to rise. This is due to a different reasoning from the above. As the right leg experiences more pain, more load is being transferred to the left leg. Therefore, the quadriceps on the left need to work harder to compensate for those load, registering a higher EMG activity. This should be taken into consideration when manufacturing PTB sockets, because it will cause faster muscle fatigue on the left due to the additional workload. A decrease in the EMG activity is observed when the PTB depth is relieved from neutral to $-4 \mathrm{~mm}$. This is because of the less pressure being exerted to the patellar tendon, hence the right quadriceps will experience less pain. As a result, the left quadriceps will have less strain, registering the same decreasing pattern.

Figures 2(a)-2(d) are graphs generated by the hip extensors and knee flexors as a result of varying PTB rectification depth. On the right hip extensors [Fig. 2(a)], 
Area under right rectus femoris EMG curve at various PTB rectification depths

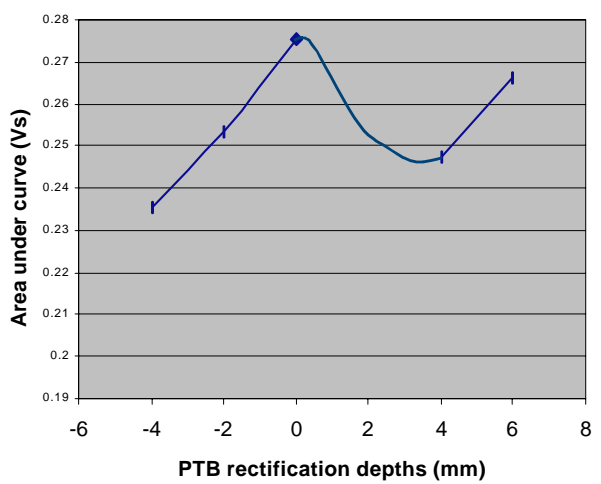

(a)

\section{Area under right vastus lateralis curve at} diffe rent PTB rectification de pths

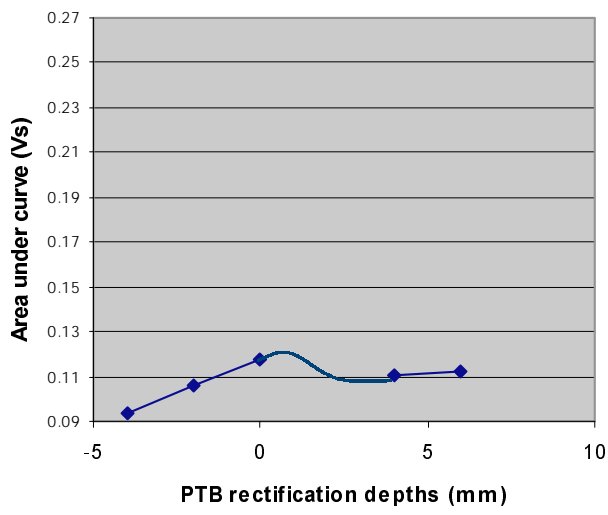

(c)
Area under left rectus femoris EMG curve at various $P$ TB rectification depths

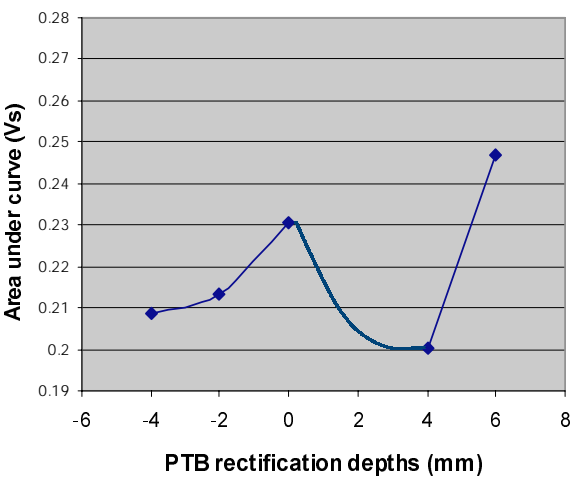

(b)

\section{Area under left vastus late ralis EMG curve at various $P T B$ rectification de pths}

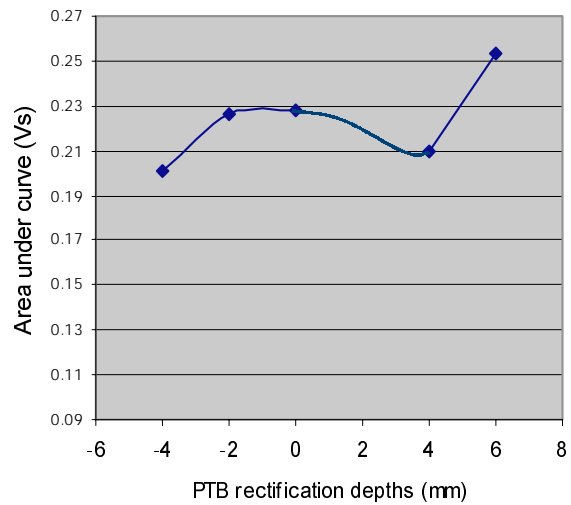

(d)

Fig. 1. Area under the (a) right rectus femoris, (b) left rectus femoris, (c) right vastus lateralis, and (d) left vastus lateralis EMG curve with the subject wearing PTB at various rectification depths.

the rectification of PTB results in an almost linear relationship from relief $-4 \mathrm{~mm}$ to $+6 \mathrm{~mm}$. This pattern, is actually similar to the pattern obtained in Figs. 1(a)1(d). However, for the right biceps femoris [Fig. 2(c)], the activity increases when the PTB is relieved from 0 to $-4 \mathrm{~mm}$. The PTB neutral experiences the minimum EMG. This is because of the design of the socket is such that more pressure is exerted on to the patellar tendon, and less on the posterior side to allow movement of the hamstring tendons. This EMG rises when the PTB is relieved from neutral 
Area under right gluteus maxim us EMG curve at different $\mathrm{PTB}$ rectification depths

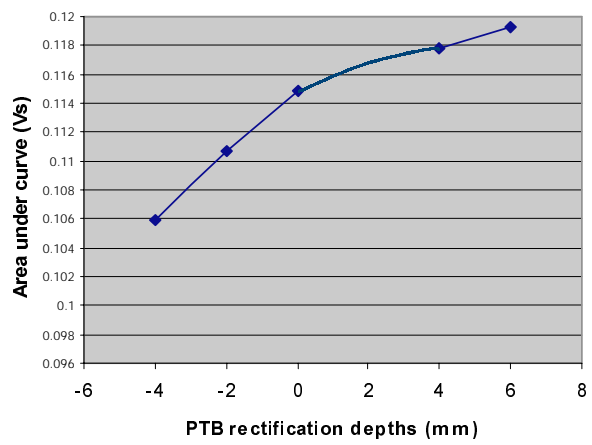

(a)

Area under right biceps femoris EMG curve at different PTB rectification depths

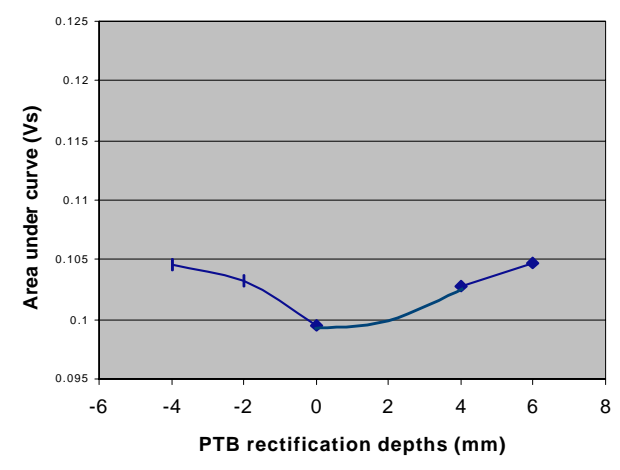

(c)
Area under left glute us maxim us EMG curve at diffe rent $P$ TB rectification depths

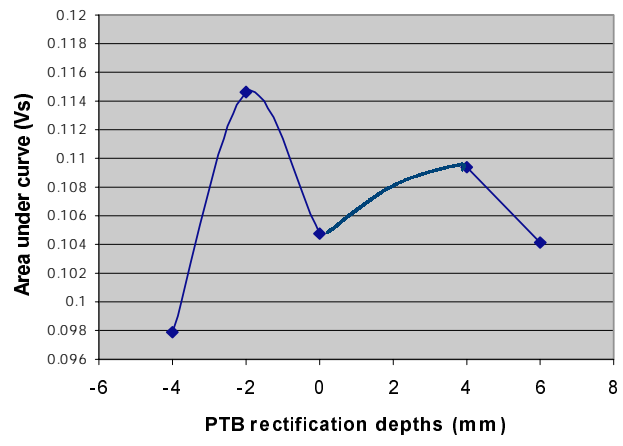

(b)

Area under left biceps femoris EMG curve at different $P$ TB rectification depths

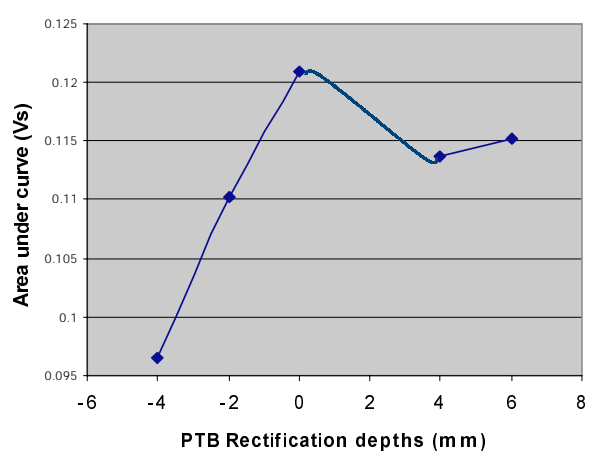

(d)

Fig. 2. Area under the (a) right gluteus maximus, (b) left gluteus maximus, (c) right bicep femoris, and (d) left bicep femoris EMG curve with the subject wearing PTB at various rectification depths.

position. This can be rationalized by the fact that as there is more room given at the patellar tendon, the stump will inevitably rest on the posterior wall of the socket, since the PTB manufactured is a total contact socket. As a result, the hamstring movement is restrained, producing higher muscle activity when flexing the knee. The left biceps femoris [Fig. 2(d)], however, will not have this problem, and the pattern generally follows that of the quadriceps groups since the quadriceps are co-contracting with the hamstrings to control excessive knee flexion. Therefore, the activities of the two groups of the muscles are similar. This also accounts for the increase in EMG for the right biceps femoris when the rectification depth is 
increased to $+6 \mathrm{~mm}$. Figure $2(\mathrm{c})$ is unique from all other graphs, because the EMG activity rises, from compression $+6 \mathrm{~mm}$ to $+4 \mathrm{~mm}$, and a peak is produced in $-2 \mathrm{~mm}$ before falling to the minimum at $-4 \mathrm{~mm}$. The minimum points at $+6 \mathrm{~mm}$ and neutral position can be rationalized by the compensation of the hip extension achieved by the left biceps femoris (since the two maximum points occurs at neutral and $+6 \mathrm{~mm}$ ). The maximum point of the left gluteus maximus EMG is at $-2 \mathrm{~mm}$, which makes up for the lower activity at the left biceps femoris. However, both the muscles registered least activity at PTB $-4 \mathrm{~mm}$. This may again, be due to the least pain experienced by the amputated leg, causing the left leg to be relieved from additional load transfer.

\section{Conclusion}

The prosthetic leg is unable to produce sufficient braking and propelling force due to the loss of plantarflexors and dorsiflexors. The surface EMG is able to detect and record the activity of the muscles during walking. By observing the pattern on the computer-processed EMG linear envelope, the contraction of these muscles to produce a coordinated, periodic walk can be known. The amputee's neural system has acknowledged his/her neural system and has compensated for the lost motor function and altered anthropometrics. This experiment has shown that the muscles of the lower extremities of an amputee, including hip extensors, hip flexors, knee extensors and knee flexors have hyperactive muscle activity during stance phase. This is to resist collapse and to extend sufficiently to achieve the required pushoff without the lower amputated limb. The rectification of the PTB at different depths can affect the EMG activities of the amputee in a certain pattern. From the results shown, more relief from the standard neutral position of $18 \mathrm{~mm}$, up to $-4 \mathrm{~mm}$ will produce less activity for most of the muscles, except the hamstrings. More rectification into the patellar tendon will cause more pain to the amputee due to the increased constraints on the quadriceps. However, it is not a direct linear relationship since the resulting curve is has an ' $N$ ' pattern. This may lead to the identification of a new, optimum PTB rectification depth, which better restores the ambulation of amputee with improved load distribution. Nonetheless, the rectification depths of a range from relief $4 \mathrm{~mm}$ to compression $6 \mathrm{~mm}$ can be tolerated by the amputee simply because the amputee can still walk in the these PTBs. This may provide a guide for inexperienced prosthetists in the manufacturing of PTB sockets.

\section{References}

[1] Murdoch, G., ed., Prosthetic and Orthotic Practice, London, Edward Arnold Ltd (1969).

[2] Abu Osman, N. A., Biomechanical measurement on stresses acting at patellar tendon bar by rectification of a PTB prosthesis socket, Proc. Nat. Conf. Biomed. Eng., Petaling Jaya, Selangor (2000), pp. 61-63. 
[3] Pinzur, M. S., Cox, W., Kaiser, J., Morris, T., Patwardhan, A. and Vrbos, L., The effect of prosthetic alignment on relative limb loading in persons with transtibial amputation: A preliminary report, J. Rehabil. Res. Dev., 32(4) (1995), pp. 373-378.

[4] Lee, V. S. P., Solomonidis, S. E. and Spence, W. D., Stump-socket interface pressure as an aid to socket design in prostheses for trans-femoral amputees - A preliminary study, Proc. Instn. Mech. Engrs., 211 (1991), pp. 167-180.

[5] Berme, N., Lawes, P., Solomonidis, S. and Paul, J. P., A shorter pylon transducer for measurement of prosthetic forces and moments during amputee gait, Engineering in Medicine, (1976), pp. 6-8.

-[6] Williams, R. B., Porter, D. and Roberts, V. C., Triaxial force transducer for investigating stresses at the stump/socket interface, Med. Biol. Eng. Comput., 30 (1992), 89-96.

-[7] Sanders, J. E., Bell, D. M., Okumura, R. M. and Dralle, A. J., Effects of alignment changes on stance phase pressures and shear stresses on transtibial amputees: Measurements from 13 transducer sites, IEEE Trans. on Rehabil. Eng., 6(1) (1998), 21-31.

[8] Peat, M., Dubo, H. I. C., Winter, D. A., Quanbury, A. O., Steinke, T. and Grahame, R., Electromyographic temporal analysis of gait: Hemiplegic locomotion, Arch. Phys. Med. Rehabil., 57 (1976), 421-425.

- [9] Winter, D. A. and Sienko, S. E.,Biomechanics of below-knee amputee gait, J. Biomech., 21(5) (1984), 361-367.

[10] Winter, D. A., Pathologic gait diagnosis with computer-averaged electromyographic profiles, Arch. Phys. Med. Rehabil., 65 (1984), 393-398.

[11] Peat, M., Dubo, H. I. C., Winter, D. A., Quanbury, A. O., Steinke, T. and Grahame, R., Electromyographic temporal analysis of gait: Hemiplegic locomotion, Arch. Phys. Med. Rehabil., 57 (1976), 421-425.

[12] Vaughn, C. L., Davis, B. L. and O'Connor, J. C., Dynamics of Human Gait, Illinois, Human Kinetics (1992).

[13] Synder, R. D., Powers, C. M., Fontaine, C. and Perry, J., The effect of five prosthetic feet on the gait and loading of the sound limb in dysvascular below-knee amputees, J. Rehabil. Res. Dev., 32(4) (1995), 309-315.

[14] Whittle, M. W., Gait Analysis: An Introduction, 2nd edn. Oxford, Butterworth Heinemann (1996).

[15] Winter, D. A., Overall principal of lower limb support during stance phase of gait, J. Biomech., 13 (1980), 923-927. 